\title{
A Compact Difference Scheme for a Class of Variable Coefficient Quasilinear Parabolic Equations with Delay
}

\author{
Wei Gu \\ School of Statistics and Mathematics, Zhongnan University of Economics and Law, Wuhan, Hubei 430073, China \\ Correspondence should be addressed to Wei Gu; wei_gu@znufe.edu.cn
}

Received 19 February 2014; Accepted 15 May 2014; Published 5 June 2014

Academic Editor: Zhongxiao Jia

Copyright (C) 2014 Wei Gu. This is an open access article distributed under the Creative Commons Attribution License, which permits unrestricted use, distribution, and reproduction in any medium, provided the original work is properly cited.

A linearized compact difference scheme is provided for a class of variable coefficient parabolic systems with delay. The unique solvability, unconditional stability, and convergence of the difference scheme are proved, where the convergence order is four in space and two in time. A numerical test is presented to illustrate the theoretical results.

\section{Introduction}

From the twentieth century, more and more scholars have been attracted into the research on the theory of delay differential equations (DDEs) [1-4]. As we know, most DDEs have no analytical solutions; efficient numerical methods solving for DDEs and delay partial differential equations (DPDEs) need to be considered deeply. Recently, many scholars consider the numerical investigation on DPDEs. For instance, Marzban and Tabrizidooz [5] considered a hybrid approximation method for solving Hutchinson's equation; Jackiewicz and Zubik-Kowal [6] considered Chebyshev spectral collocation and waveform relaxation methods for nonlinear DPDEs and finite difference methods were considered to solve delay parabolic partial differential equations in [79]; Li et al. [10-12] constructed finite element methods to solve reaction-diffusion equations with delay. The numerical research of DPDEs focused on stability analysis can be referred to in [13].

The following variable coefficient parabolic systems with delay are considered in this paper:

$$
\begin{array}{r}
r(x, t) u_{t}-d u_{x x}=f(u(x, t), u(x, t-s), x, t), \\
(x, t) \in(0,1) \times(0, T],
\end{array}
$$

$$
\begin{gathered}
u(x, t)=\phi(x, t), \quad x \in[0,1], t \in[-s, 0], \\
u(0, t)=\alpha(t), \quad u(1, t)=\beta(t), \quad t \in(0, T],
\end{gathered}
$$

where $d>0$ is a constant and $s>0$ is the delay term, $r(x, t) \in C((0,1) \times(0, T]), 0<c_{0} \leq r(x, t) \leq c_{1}$. In the special case of $r(x, t)=1$, numerical solutions of (1)-(3) have been considered in [14-17]. Ferreira and da Silva considered a backward Euler scheme and proved the stability and convergence by the energy method in [14]. A Crank-Nicolson scheme and a linearized compact difference scheme were proposed by Zhang and Sun in [15] and Sun and Zhang in [16], respectively. Q. Zhang and C. Zhang considered a new linearized compact multisplitting scheme in [17]. Gu and Wang constructed a Crank-Nicolson scheme in [18] to solve a special case of (1), where $f=f(u(x, t-$ $s)$ ). In this paper, a linearized compact difference scheme solving for (1)-(3) will be constructed. The unique solvability, unconditional stability, and convergence of the difference scheme are proved, where the convergence order is four in space and two in time. A numerical test is presented to illustrate the theoretical results.

The paper is organized as follows. In Section 2, a linearized compact difference scheme is constructed to solve (1)-(3). Section 3 considers the solvability, stability, and convergence of the provided difference scheme. 
In Section 4, a numerical test is presented to illustrate the theoretical results. Section 5 gives a brief discussion of this paper.

\section{The Compact Difference Scheme and Local Truncation Error}

Throughout this paper, the following assumptions are assumed to be true.

(H1) Let $m$ be an integer satisfying $m s \leq T<(m+1) s$, denote $I_{l}=(l s, l s+s), l=-1,0, \ldots, m-1, I_{m}=$ $(m s, T)$, and $I=\cup_{p=-1}^{m} I_{p}$, assume that (1)-(3) has a unique solution $u \in C^{6,4}(I \times(0, T])$ and that $u$ and its partial derivatives are all bounded by a constant $c_{2}$;

(H2) $f(\mu, \nu, x, t)$ has bounded first-order continuous partial derivatives, and we denote

$$
\begin{aligned}
& c_{3}=\max _{\left|\varepsilon_{1}\right| \leq \varepsilon_{0},\left|\varepsilon_{2}\right| \leq \varepsilon_{0}}\left|f_{\mu}\left(u(x, t)+\varepsilon_{1}, u(x, t-s)+\varepsilon_{2}, x, t\right)\right|, \\
& c_{4}=\max _{\left|\varepsilon_{1}\right| \leq \varepsilon_{0},\left|\varepsilon_{2}\right| \leq \varepsilon_{0}}\left|f_{v}\left(u(x, t)+\varepsilon_{1}, u(x, t-s)+\varepsilon_{2}, x, t\right)\right|,
\end{aligned}
$$

where $\varepsilon_{0}>0, c_{3}$, and $c_{4}$ are constants, $(x, t) \in(0,1) \times$ $(0, T]$.

First let $M$ and $j$ be two positive integers; then, we take $h=1 / M, \tau=s / j, x_{i}=i h, t_{k}=k \tau, t_{k+1 / 2}=\left(t_{k}+t_{k+1}\right) / 2$. Define $\Omega_{h \tau}=\Omega_{h} \times \Omega_{\tau}$, where $\Omega_{h}=\left\{x_{i} \mid 0 \leq i \leq M\right\}$, $\Omega_{\tau}=\left\{t_{k} \mid-j \leq k \leq N\right\}, N=[T / \tau]$. Denote $U_{i}^{k}=u\left(x_{i}, t_{k}\right)$, $0 \leq i \leq M,-j \leq k \leq N$, throughout this paper. Let

$$
\mathscr{W}=\left\{v_{i}^{k} \mid 0 \leq i \leq M,-j \leq k \leq N\right\}
$$

be the grid function space defined on $\Omega_{h \tau}$. The following notations are made:

$$
\begin{gathered}
v_{i}^{k+(1 / 2)}=\frac{v_{i}^{k}+v_{i}^{k+1}}{2}, \\
\delta_{t} v_{i}^{k+(1 / 2)}=\frac{v_{i}^{k+1}-v_{i}^{k}}{\tau}, \\
\delta_{x} v_{i+(1 / 2)}^{k}=\frac{v_{i+1}^{k}-v_{i}^{k}}{h}, \\
\delta_{x}^{2} v_{i}^{k}=\frac{v_{i+1}^{k}-2 v_{i}^{k}+v_{i-1}^{k}}{h^{2}}, \\
\mathscr{A} v_{i}^{k}=\frac{1}{12}\left(v_{i-1}^{k}+10 v_{k}^{k}+v_{i+1}^{k}\right) .
\end{gathered}
$$

Considering (1) at the point $\left(x_{i}, t_{k+(1 / 2)}\right)$, we have

$$
\begin{array}{r}
r\left(x_{i}, t_{k+(1 / 2)}\right) \frac{\partial u}{\partial t}\left(x_{i}, t_{k+(1 / 2)}\right)-d \frac{\partial^{2} u}{\partial x^{2}}\left(x_{i}, t_{k+(1 / 2)}\right) \\
=f\left(u\left(x_{i}, t_{k+(1 / 2)}\right), u\left(x_{i}, t_{k+(1 / 2)-j}\right), x_{i}, t_{k+(1 / 2)}\right), \\
0 \leq i \leq M, 0 \leq k \leq N-1 .
\end{array}
$$

From Taylor expansion, we have

$$
\begin{array}{r}
\frac{\partial u}{\partial t}\left(x_{i}, t_{k+(1 / 2)}\right)=\delta_{t} U_{i}^{k+(1 / 2)}-\frac{\tau^{2}}{24} \frac{\partial^{3} u}{\partial t^{3}}\left(x_{i}, \eta_{i}^{k}\right), \\
\eta_{i}^{k} \in\left(t_{k}, t_{k+1}\right),
\end{array}
$$

$$
\begin{aligned}
\frac{\partial^{2} u}{\partial x^{2}}\left(x_{i}, t_{k+(1 / 2)}\right) & \\
= & \frac{1}{2}\left[\frac{\partial^{2} u}{\partial x^{2}}\left(x_{i}, t_{k}\right)+\frac{\partial^{2} u}{\partial x^{2}}\left(x_{i}, t_{k+1}\right)\right] \\
& -\frac{\tau^{2}}{8} \frac{\partial^{4} u}{\left.\partial x^{2} \partial t^{2}\right)}\left(x_{i}, \gamma_{i}^{k}\right) \\
= & \frac{1}{2}\left(\delta_{x}^{2} U_{i}^{k}+\delta_{x}^{2} U_{i}^{k+1}\right) \\
& -\frac{h^{2}}{24}\left[\frac{\partial^{4} u}{\partial x^{4}}\left(\xi_{i}^{k}, t_{k}\right)+\frac{\partial^{4} u}{\partial x^{4}}\left(\xi_{i}^{k+1}, t_{k+1}\right)\right] \\
& -\frac{\tau^{2}}{8} \frac{\partial^{4} u}{\partial x^{2} \partial t^{2}}\left(x_{i}, \gamma_{i}^{k}\right),
\end{aligned}
$$

$$
\begin{aligned}
\xi_{i}^{k}, \xi_{i}^{k+1} \in\left(x_{i-1}, x_{i+1}\right), \quad \gamma_{i}^{k} \in\left(t_{k}, t_{k+1}\right), \\
f\left(u\left(x_{i}, t_{k+(1 / 2)}\right), u\left(x_{i}, t_{k+(1 / 2)-j}\right), x_{i}, t_{k+(1 / 2)}\right) \\
=f\left(\frac{3}{2} U_{i}^{k}-\frac{1}{2} U_{i}^{k-1}, \frac{1}{2} U_{i}^{k+1-j}+\frac{1}{2} U_{i}^{k-j}, x_{i}, t_{k+(1 / 2)}\right) \\
\quad+\frac{3 \tau^{2}}{8} \frac{\partial^{2} u\left(x_{i}, \rho^{k}\right)}{\partial t^{2}} f_{\mu}\left(\zeta_{i}^{k}, \varsigma_{i}^{k}, x_{i}, t_{k+(1 / 2)}\right) \\
\quad-\frac{\tau^{2}}{8} \frac{\partial^{2} u\left(x_{i}, \varrho^{k}\right)}{\partial t^{2}} f_{\nu}\left(\zeta_{i}^{k}, \varsigma_{i}^{k}, x_{i}, t_{k+(1 / 2)}\right),
\end{aligned}
$$

where $\rho^{k} \in\left(t_{k-1}, t_{k+1 / 2}\right), \varrho^{k} \in\left(t_{k-j}, t_{k+1-j}\right), \zeta_{i}^{k}$ is between $u\left(x_{i}, t_{k+(1 / 2)}\right)$ and $(3 / 2) U_{i}^{k}-(1 / 2) U_{i}^{k-1}$, and $\varsigma_{i}^{k}$ is between $u\left(x_{i}, t_{k+(1 / 2)-j}\right)$ and $(1 / 2) U_{i}^{k+1-j}+(1 / 2) U_{i}^{k-j}$. Substituting (8) into (7), denote $r_{i}^{k+(1 / 2)}=r\left(x_{i}, t_{k+(1 / 2)}\right)$; we obtain

$$
\begin{gathered}
r_{i}^{k+(1 / 2)} \delta_{t} U_{i}^{k+(1 / 2)}-\frac{d}{2}\left[\frac{\partial^{2} u}{\partial x^{2}}\left(x_{i}, t_{k}\right)+\frac{\partial^{2} u}{\partial x^{2}}\left(x_{i}, t_{k+1}\right)\right] \\
=f\left(\frac{3}{2} U_{i}^{k}-\frac{1}{2} U_{i}^{k-1}, \frac{1}{2} U_{i}^{k+1-j}+\frac{1}{2} U_{i}^{k-j}, x_{i}, t_{k+(1 / 2)}\right) \\
+\tau^{2} \bar{R}_{i}^{k}, \quad 1 \leq i \leq M, 0 \leq k \leq N-1,
\end{gathered}
$$

where

$$
\begin{aligned}
\bar{R}_{i}^{k}= & \frac{r_{i}^{k+(1 / 2)}}{24} \frac{\partial^{3} u}{\partial t^{3}}\left(x_{i}, \eta_{i}^{k}\right)-\frac{d}{8} \frac{\partial^{4} u}{\partial x^{2} \partial t^{2}}\left(x_{i}, \gamma_{i}^{k}\right) \\
& +\frac{3}{8} \frac{\partial^{2} u\left(x_{i}, \rho^{k}\right)}{\partial t^{2}} f_{\mu}\left(\zeta_{i}^{k}, \varsigma_{i}^{k}, x_{i}, t_{k+(1 / 2)}\right) \\
& -\frac{1}{8} \frac{\partial^{2} u\left(x_{i}, \varrho^{k}\right)}{\partial t^{2}} f_{\nu}\left(\zeta_{i}^{k}, \varsigma_{i}^{k}, x_{i}, t_{k+(1 / 2)}\right) .
\end{aligned}
$$


Acting operator $\mathscr{A}$ on both sides of (9), we have

$$
\begin{aligned}
& \mathscr{A} r_{i}^{k+(1 / 2)} \delta_{t} U_{i}^{k+(1 / 2)} \\
& -\frac{d}{2}\left[\mathscr{A} \frac{\partial^{2} u}{\partial x^{2}}\left(x_{i}, t_{k}\right)+\mathscr{A} \frac{\partial^{2} u}{\partial x^{2}}\left(x_{i}, t_{k+1}\right)\right] \\
& =\mathscr{A} f\left(\frac{3}{2} U_{i}^{k}-\frac{1}{2} U_{i}^{k-1}, \frac{1}{2} U_{i}^{k+1-j}+\frac{1}{2} U_{i}^{k-j}, x_{i}, t_{k+(1 / 2)}\right) \\
& +\tau^{2} \mathscr{A} \bar{R}_{i}^{k}, \quad 1 \leq i \leq M-1,0 \leq k \leq N-1 .
\end{aligned}
$$

Resorting to the following Lemma, we can obtain the estimation of the operator $\mathscr{A}$.

Lemma 1 (see $[19,20]$ ). Suppose that $q(x) \in C^{6}\left[x_{i-1}, x_{i+1}\right]$; then, we have

$$
\begin{aligned}
\frac{1}{12} & {\left[q^{\prime \prime}\left(x_{i-1}\right)+10 q^{\prime \prime}\left(x_{i}\right)+q^{\prime \prime}\left(x_{i+1}\right)\right] } \\
& -\frac{1}{h^{2}}\left[q\left(x_{i-1}\right)-2 q\left(x_{i}\right)+q\left(x_{i+1}\right)\right] \\
= & \frac{h^{4}}{240} q^{(6)}\left(\omega_{i}\right),
\end{aligned}
$$

where $\omega_{i} \in\left(x_{i-1}, x_{i+1}\right)$.

From Lemma 1 and Taylor expansion, we obtain

$$
\begin{gathered}
\mathscr{A} \frac{\partial^{2} u}{\partial x^{2}}\left(x_{i}, t_{k}\right)=\delta_{x}^{2} U_{i}^{k}+\frac{h^{4}}{240} \frac{\partial^{6} u}{\partial x^{6}}\left(\theta_{i}^{k}, t_{k}\right), \\
\theta_{i}^{k} \in\left(x_{i-1}, x_{i+1}\right), \\
\mathscr{A} \frac{\partial^{2} u}{\partial x^{2}}\left(x_{i}, t_{k+1}\right)=\delta_{x}^{2} U_{i}^{k+1}+\frac{h^{4}}{240} \frac{\partial^{6} u}{\partial x^{6}}\left(\theta_{i}^{k+1}, t_{k+1}\right), \\
\theta_{i}^{k+1} \in\left(x_{i-1}, x_{i+1}\right) .
\end{gathered}
$$

Inserting (13) into (11), we have

$$
\begin{aligned}
& \mathscr{A} r_{i}^{k+(1 / 2)} \delta_{t} U_{i}^{k+(1 / 2)}-d \delta_{x}^{2} U_{i}^{k+1 / 2} \\
& =\mathscr{A} f\left(\frac{3}{2} U_{i}^{k}-\frac{1}{2} U_{i}^{k-1}, \frac{1}{2} U_{i}^{k+1-j}+\frac{1}{2} U_{i}^{k-j}, x_{i}, t_{k+(1 / 2)}\right) \\
& \quad+R_{i}^{k}, \quad 1 \leq i \leq M-1,0 \leq k \leq N-1,
\end{aligned}
$$

where

$$
R_{i}^{k}=\tau^{2} \mathscr{A} \bar{R}_{i}^{k}+\frac{d h^{4}}{480}\left[\frac{\partial^{6} u}{\partial x^{6}}\left(\theta_{i}^{k}, t_{k}\right)+\frac{\partial^{6} u}{\partial x^{6}}\left(\theta_{i}^{k+1}, t_{k+1}\right)\right] .
$$

From $0<c_{0} \leq r_{i}^{k+1 / 2} \leq c_{1}$ and assumptions (H1) and (H2), we have

$$
\left|\bar{R}_{i}^{k}\right| \leq c_{5}, \quad 1 \leq i \leq M-1,0 \leq k \leq N-1,
$$

such that

$$
\left|R_{i}^{k}\right| \leq c_{6}\left(\tau^{2}+h^{4}\right), \quad 1 \leq i \leq M-1,0 \leq k \leq N-1 .
$$

Discretizing the initial and boundary conditions of (2) and (3), we obtain

$$
\begin{gathered}
U_{i}^{k}=\phi\left(x_{i}, t_{k}\right), \quad 0 \leq i \leq M,-j \leq k \leq 0, \\
U_{0}^{k}=\alpha\left(t_{k}\right), \quad U_{M}^{k}=\beta\left(t_{k}\right), \quad 1 \leq k \leq N .
\end{gathered}
$$

Replacing $U_{i}^{k}$ by $u_{i}^{k}$ in (14) and omitting $R_{i}^{k}$, we obtain the following compact difference scheme:

$$
\begin{gathered}
\mathscr{A} r_{i}^{k+(1 / 2)} \delta_{t} u_{i}^{k+(1 / 2)}-d \delta_{x}^{2} u_{i}^{k+1 / 2} \\
=\mathscr{A} f\left(\frac{3}{2} u_{i}^{k}-\frac{1}{2} u_{i}^{k-1}, \frac{1}{2} u_{i}^{k+1-j}+\frac{1}{2} u_{i}^{k-j}, x_{i}, t_{k+(1 / 2)}\right), \\
1 \leq i \leq M-1,0 \leq k \leq N-1, \\
u_{i}^{k}=\phi\left(x_{i}, t_{k}\right), \quad 0 \leq i \leq M,-j \leq k \leq 0 \\
u_{0}^{k}=\alpha\left(t_{k}\right), \quad u_{M}^{k}=\beta\left(t_{k}\right), \quad 1 \leq k \leq N .
\end{gathered}
$$

\section{The Solvability, Convergence, and Stability of the Compact Difference Scheme}

Define the following grid function space on $\Omega_{h}$ :

$$
V=\left\{v \mid v=\left(v_{0}, v_{1}, \ldots, v_{M}\right), v_{0}=v_{M}=0\right\} .
$$

If $v \in V$, we introduce the following notations:

$$
\begin{aligned}
&\|v\|=\sqrt{h \sum_{i=1}^{M-1}\left(v_{i}\right)^{2},} \\
&|v|_{1}=\sqrt{h \sum_{i=1}^{M}\left(\frac{v_{i}-v_{i-1}}{h}\right)^{2}}, \\
&\|v\|_{\infty}=\max _{0 \leq i \leq M}\left|v_{i}\right| .
\end{aligned}
$$

By $[16,17,19]$, we have the following two inequalities:

$$
\begin{aligned}
\|v\|_{\infty} & \leq \frac{1}{2}|v|_{1}, \\
\|v\| & \leq \frac{1}{\sqrt{6}}|v|_{1} .
\end{aligned}
$$

For the analysis of the difference scheme, the following Lemma is introduced.

Lemma 2 (see $[16,17,19]$ ). Assume that $\left\{F^{k} \mid k \geq 0\right\}$ to be nonnegative sequence and satisfies

$$
F^{k+1} \leq A+B \tau \sum_{i=1}^{k} F^{l}, \quad k=0,1, \ldots
$$

then

$$
F^{k+1} \leq A \exp (B k \tau), \quad k=0,1,2, \ldots,
$$

where $A$ and $B$ are nonnegative constants. 
Theorem 3. Under the condition that $5 c_{0}-c_{1}>0$, the compact difference scheme (20)-(22) has a unique solution.

Proof. Denote that $\lambda=d \tau / h^{2}$; then, difference scheme (20)(22) can be reformed as

$$
\begin{aligned}
& \left(\frac{1}{12} r_{i-1}^{k+1 / 2}-\frac{\lambda}{2}\right) u_{i-1}^{k+1}+\left(\frac{10}{12} r_{i}^{k+1 / 2}+\lambda\right) u_{i}^{k+1} \\
& +\left(\frac{1}{12} r_{i+1}^{k+1 / 2}-\frac{\lambda}{2}\right) u_{i+1}^{k+1} \\
& =\left(\frac{1}{12} r_{i-1}^{k+1 / 2}+\frac{\lambda}{2}\right) u_{i-1}^{k} \\
& +\left(\frac{10}{12} r_{i}^{k+1 / 2}-\lambda\right) u_{i}^{k}+\left(\frac{1}{12} r_{i+1}^{k+1 / 2}+\frac{\lambda}{2}\right) u_{i+1}^{k} \\
& +\frac{\tau}{12} f\left(\frac{3}{2} u_{i-1}^{k}-\frac{1}{2} u_{i-1}^{k-1}, \frac{1}{2} u_{i-1}^{k+1-j}\right. \\
& \left.\quad+\frac{1}{2} u_{i-1}^{k-j}, x_{i-1}, t_{k+(1 / 2)}\right) \\
& +\frac{10 \tau}{12} f\left(\frac{3}{2} u_{i}^{k}-\frac{1}{2} u_{i}^{k-1}, \frac{1}{2} u_{i}^{k+1-j}\right. \\
& \quad+\frac{\tau}{12} f\left(\frac{3}{2} u_{i+1}^{k}-\frac{1}{2} u_{i+1}^{k-j}, x_{i}, t_{k+(1 / 2)}\right) \\
& \left.+\frac{1}{2} u_{i+1}^{k-j}, x_{i+1}, t_{k+(1 / 2)}^{k+1-j}\right) \\
& +
\end{aligned}
$$

The mathematical induction method will be used in the proof of this theorem. Denote

$$
u^{k}=\left(u_{0}^{k}, u_{1}^{k}, \ldots, u_{M}^{k}\right)
$$

Notice that $u^{k}(-j \leq k \leq 0)$ is determined by the initial condition (21). Suppose that $u^{l}$ has been determined.

Let $k=l$ in (20); the linear algebraic equations with respect to $u^{l+1}$ can be obtained. Under the condition that $5 c_{0}-c_{1}>0$, we have

$$
\begin{aligned}
& \left|\frac{10}{12} r_{i}^{l+1 / 2}+\lambda\right|-\left|\frac{1}{12} r_{i-1}^{l+1 / 2}-\frac{\lambda}{2}\right|-\left|\frac{1}{12} r_{i+1}^{l+1 / 2}-\frac{\lambda}{2}\right| \\
& \geq \frac{10}{12} r_{i}^{l+1 / 2}-\frac{1}{12} r_{i-1}^{l+1 / 2}-\frac{1}{12} r_{i+1}^{l+1 / 2} \\
& \geq \frac{10 c_{0}-2 c_{1}}{12} \\
& >0 .
\end{aligned}
$$

Thus, the coefficient matrix of the linear algebraic system is strictly diagonally dominant and then there exists a unique solution $u^{l+1}$. By the inductive principle, the proof ends.
Denote $e_{i}^{k}=U_{i}^{k}-u_{i}^{k}, 0 \leq i \leq M,-j \leq k \leq N$; subtracting (20)-(22) from (14), (18), and (19), respectively, the following error equations can be obtained:

$$
\begin{gathered}
\mathscr{A} r_{i}^{k+(1 / 2)} \delta_{t} e_{i}^{k+(1 / 2)}-d \delta_{x}^{2} e_{i}^{k+1 / 2} \\
=\mathscr{A}\left[f\left(\frac{3}{2} U_{i}^{k}-\frac{1}{2} U_{i}^{k-1}, \frac{1}{2} U_{i}^{k+1-j}+\frac{1}{2} U_{i}^{k-j}, x_{i}, t_{k+(1 / 2)}\right)\right. \\
\left.\quad-f\left(\frac{3}{2} u_{i}^{k}-\frac{1}{2} u_{i}^{k-1}, \frac{1}{2} u_{i}^{k+1-j}+\frac{1}{2} u_{i}^{k-j}, x_{i}, t_{k+(1 / 2)}\right)\right] \\
+R_{i}^{k}, \quad 1 \leq i \leq M-1,0 \leq k \leq N-1 \\
e_{i}^{k}=0, \quad 0 \leq i \leq M,-j \leq k \leq 0 \\
e_{0}^{k}=0, \quad e_{M}^{k}=0, \quad 1 \leq k \leq N .
\end{gathered}
$$

Theorem 4. Denote

$$
C=c_{6} \sqrt{\frac{3 T}{d\left(9 c_{0}-c_{1}\right)}} \exp \left(\frac{2\left(5 c_{3}^{2}+c_{4}^{2}\right)}{d\left(9 c_{0}-c_{1}\right)} T\right) .
$$

If the following conditions are satisfied:

$$
\tau \leq\left(\frac{\epsilon_{0}}{4 C}\right)^{1 / 2}, \quad h \leq\left(\frac{\epsilon_{0}}{4 C}\right)^{1 / 4},
$$

then we have

$$
\left\|e^{k}\right\|_{\infty} \leq C\left(\tau^{2}+h^{4}\right), \quad 0 \leq k \leq N,
$$

where $\epsilon_{0}>0$ is a constant.

Proof. Acting $h \delta_{t} e_{i}^{k+(1 / 2)}$ on (32) and summing up for $i$ from 1 to $M-1$, we obtain

$$
\begin{aligned}
& h \sum_{i=1}^{M-1}\left(\mathscr{A} r_{i}^{k+(1 / 2)} \delta_{t} e_{i}^{k+(1 / 2)}\right) \delta_{t} e_{i}^{k+(1 / 2)} \\
& \quad-d h \sum_{i=1}^{M-1}\left(\delta_{x}^{2} e_{i}^{k+1 / 2}\right) \delta_{t} e_{i}^{k+(1 / 2)} \\
& =h \sum_{i=1}^{M-1}\left\{\mathscr { A } \left[f \left(\frac{3}{2} U_{i}^{k}-\frac{1}{2} U_{i}^{k-1}, \frac{1}{2} U_{i}^{k+1-j}\right.\right.\right. \\
& \left.\quad+\frac{1}{2} U_{i}^{k-j}, x_{i}, t_{k+(1 / 2)}\right) \\
& \quad f\left(\frac{3}{2} u_{i}^{k}-\frac{1}{2} u_{i}^{k-1}, \frac{1}{2} u_{i}^{k+1-j}\right. \\
& \left.\left.\left.\quad+\frac{1}{2} u_{i}^{k-j}, x_{i}, t_{k+(1 / 2)}\right)\right]\right\} \delta_{t} e_{i}^{k+(1 / 2)} \\
& +h \sum_{i=1}^{M-1}\left(R_{i}^{k}\right) \delta_{t} e_{i}^{k+(1 / 2)}, \quad 1 \leq i \leq M-1,0 \leq k \leq N-1 .
\end{aligned}
$$

Mathematical induction will be used to prove this theorem. Notice that $\left\|e^{k}\right\|_{\infty}=0(-j \leq k \leq 0)$ and suppose that 
(37) is true for $0 \leq k \leq l$; we will show that (37) is also true for $k=l+1$.

In the following, each term of (38) will be estimated:

$$
\begin{aligned}
& h \sum_{i=1}^{M-1}\left(A r_{i}^{k+(1 / 2)} \delta_{t} e_{i}^{k+(1 / 2)}\right) \delta_{t} e_{i}^{k+(1 / 2)} \\
& \geq \frac{h}{12} \sum_{i=1}^{M-1}\left[9 r_{i}^{k+(1 / 2)}-\frac{\left(r_{i-1}^{k+(1 / 2)}+r_{i+1}^{k+(1 / 2)}\right)}{2}\right]\left(\delta_{t} e_{i}^{k+(1 / 2)}\right)^{2} \\
& \geq \frac{9 c_{0}-c_{1}}{12}\left\|\delta_{t} e^{k+(1 / 2)}\right\|^{2}, \\
& -d h \sum_{i=1}^{M-1}\left(\delta_{x}^{2} e_{i}^{k+(1 / 2)}\right) \delta_{t} e_{i}^{k+(1 / 2)}=\frac{d}{2 \tau}\left(\left|e^{k+1}\right|_{1}^{2}-\left|e^{k}\right|_{1}^{2}\right) \\
& h \sum_{i=1}^{M-1}\left(R_{i}^{k}\right) \delta_{t} e_{i}^{k+(1 / 2)} \\
& \leq \frac{6}{9 c_{0}-c_{1}} h \sum_{i=1}^{M-1}\left(R_{i}^{k}\right)^{2}+\frac{9 c_{0}-c_{1}}{24} h \sum_{i=1}^{M-1}\left(\delta_{t} e_{i}^{k+(1 / 2)}\right)^{2} \\
& \leq \frac{6}{9 c_{0}-c_{1}} c_{6}^{2}\left(\tau^{2}+h^{4}\right)^{2}+\frac{9 c_{0}-c_{1}}{24}\left\|\delta_{t} e^{k+(1 / 2)}\right\|^{2}
\end{aligned}
$$

From the inductive assumption and (36), we have

$$
\left\|e^{k}\right\|_{\infty} \leq C\left(\tau^{2}+h^{4}\right) \leq \frac{\epsilon_{0}}{2}, \quad 0 \leq k \leq l
$$

From (H2), we have

$$
\begin{gathered}
\mid f\left(\frac{3}{2} U_{i}^{k}-\frac{1}{2} U_{i}^{k-1}, \frac{1}{2} U_{i}^{k+1-j}+\frac{1}{2} U_{i}^{k-j}, x_{i}, t_{k+(1 / 2)}\right) \\
-f\left(\frac{3}{2} u_{i}^{k}-\frac{1}{2} u_{i}^{k-1}, \frac{1}{2} u_{i}^{k+1-j}+\frac{1}{2} u_{i}^{k-j}, x_{i}, t_{k+(1 / 2)}\right) \mid \\
\leq c_{3}\left|\frac{3}{2} e_{i}^{k}-\frac{1}{2} e_{i}^{k-1}\right|+c_{4}\left|\frac{1}{2} e_{i}^{k+1-j}+\frac{1}{2} e_{i}^{k-j}\right|, \\
1 \leq i \leq M, 0 \leq k \leq l .
\end{gathered}
$$

It then follows that

$$
\begin{gathered}
\mid \mathscr{A}\left[f\left(\frac{3}{2} U_{i}^{k}-\frac{1}{2} U_{i}^{k-1}, \frac{1}{2} U_{i}^{k+1-j}+\frac{1}{2} U_{i}^{k-j}, x_{i}, t_{k+(1 / 2)}\right)\right. \\
\left.-f\left(\frac{3}{2} u_{i}^{k}-\frac{1}{2} u_{i}^{k-1}, \frac{1}{2} u_{i}^{k+1-j}+\frac{1}{2} u_{i}^{k-j}, x_{i}, t_{k+(1 / 2)}\right)\right] \mid \\
\leq \mathscr{A}\left(c_{3}\left|\frac{3}{2} e_{i}^{k}-\frac{1}{2} e_{i}^{k-1}\right|+c_{4}\left|\frac{1}{2} e_{i}^{k+1-j}+\frac{1}{2} e_{i}^{k-j}\right|\right), \\
1 \leq i \leq M-1,0 \leq k \leq l .
\end{gathered}
$$

From the inequality above, we obtain

$$
\begin{aligned}
& h \sum_{i=1}^{M-1}\left\{\mathscr { A } \left[f\left(\frac{3}{2} U_{i}^{k}-\frac{1}{2} U_{i}^{k-1}, \frac{1}{2} U_{i}^{k+1-j}+\frac{1}{2} U_{i}^{k-j}, x_{i}, t_{k+(1 / 2)}\right)\right.\right. \\
& \left.\left.-f\left(\frac{3}{2} u_{i}^{k}-\frac{1}{2} u_{i}^{k-1}, \frac{1}{2} u_{i}^{k+1-j}+\frac{1}{2} u_{i}^{k-j}, x_{i}, t_{k+(1 / 2)}\right)\right]\right\} \\
& \times \delta_{t} e_{i}^{k+(1 / 2)} \\
& \leq h \sum_{i=1}^{M-1}\left\{\mathscr{A}\left(c_{3}\left|\frac{3}{2} e_{i}^{k}-\frac{1}{2} e_{i}^{k-1}\right|+c_{4}\left|\frac{1}{2} e_{i}^{k+1-j}+\frac{1}{2} e_{i}^{k-j}\right|\right)\right\} \\
& \times \delta_{t} e_{i}^{k+(1 / 2)} \\
& \leq \frac{6}{9 c_{0}-c_{1}} h \\
& \times \sum_{i=1}^{M-1}\left\{\mathscr{A}\left(c_{3}\left|\frac{3}{2} e_{i}^{k}-\frac{1}{2} e_{i}^{k-1}\right|+c_{4}\left|\frac{1}{2} e_{i}^{k+1-j}+\frac{1}{2} e_{i}^{k-j}\right|\right)\right\}^{2} \\
& +\frac{9 c_{0}-c_{1}}{24}\left\|\delta_{t} e_{i}^{k+(1 / 2)}\right\|^{2} \\
& \leq \frac{6}{9 c_{0}-c_{1}} h \\
& \times \sum_{i=1}^{M-1}\left(c_{3}\left|\frac{3}{2} e_{i}^{k}-\frac{1}{2} e_{i}^{k-1}\right|+c_{4}\left|\frac{1}{2} e_{i}^{k+1-j}+\frac{1}{2} e_{i}^{k-j}\right|\right)^{2} \\
& +\frac{9 c_{0}-c_{1}}{24}\left\|\delta_{t} e^{k+(1 / 2)}\right\|^{2} \\
& \leq \frac{12}{9 c_{0}-c_{1}} \\
& \times\left[c_{3}^{2} h \sum_{i=1}^{M-1}\left(\frac{3}{2} e_{i}^{k}-\frac{1}{2} e_{i}^{k-1}\right)^{2}\right. \\
& \left.+c_{4}^{2} h \sum_{i=1}^{M-1}\left(\frac{1}{2} e_{i}^{k+1-j}+\frac{1}{2} e_{i}^{k-j}\right)^{2}\right] \\
& +\frac{9 c_{0}-c_{1}}{24}\left\|\delta_{t} e^{k+(1 / 2)}\right\|^{2} \\
& \leq \frac{12}{9 c_{0}-c_{1}} \\
& \times\left[\frac{5}{2} c_{3}^{2} h \sum_{i=1}^{M-1}\left(\left(e_{i}^{k}\right)^{2}+\left(e_{i}^{k-1}\right)^{2}\right)\right. \\
& \left.+\frac{1}{2} c_{4}^{2} h \sum_{i=1}^{M-1}\left(\left(e_{i}^{k+1-j}\right)^{2}+\left(e_{i}^{k-j}\right)^{2}\right)\right] \\
& +\frac{9 c_{0}-c_{1}}{24}\left\|\delta_{t} e^{k+(1 / 2)}\right\|^{2} \\
& =\frac{30}{9 c_{0}-c_{1}} c_{3}^{2}\left(\left\|e^{k}\right\|^{2}+\left\|e^{k-1}\right\|^{2}\right)
\end{aligned}
$$




$$
\begin{aligned}
& +\frac{6}{9 c_{0}-c_{1}} c_{4}^{2}\left(\left\|e^{k+1-j}\right\|^{2}+\left\|e^{k-j}\right\|^{2}\right) \\
& +\frac{9 c_{0}-c_{1}}{24}\left\|\delta_{t} e^{k+(1 / 2)}\right\|^{2}, \quad 0 \leq k \leq l .
\end{aligned}
$$

Inserting (39)-(43) into (38), we obtain

$$
\begin{aligned}
\frac{d}{2 \tau}\left(\left|e^{k+1}\right|_{1}^{2}-\left|e^{k}\right|_{1}^{2}\right) & \\
\leq & \frac{30}{9 c_{0}-c_{1}} c_{3}^{2}\left(\left\|e^{k}\right\|^{2}+\left\|e^{k-1}\right\|^{2}\right) \\
& +\frac{6}{9 c_{0}-c_{1}} c_{4}^{2}\left(\left\|e^{k+1-j}\right\|^{2}+\left\|e^{k-j}\right\|^{2}\right) \\
& +\frac{6}{9 c_{0}-c_{1}} c_{6}^{2}\left(\tau^{2}+h^{4}\right)^{2}, \quad 0 \leq k \leq l .
\end{aligned}
$$

The above inequality has the following form:

$$
\begin{aligned}
\left|e^{k+1}\right|_{1}^{2} \leq & \left|e^{k}\right|_{1}^{2}+\frac{2 \tau}{d} \frac{30}{9 c_{0}-c_{1}} c_{3}^{2}\left(\left\|e^{k}\right\|^{2}+\left\|e^{k-1}\right\|^{2}\right) \\
& +\frac{2 \tau}{d} \frac{6}{9 c_{0}-c_{1}} c_{4}^{2}\left(\left\|e^{k+1-j}\right\|^{2}+\left\|e^{k-j}\right\|^{2}\right) \\
& +\frac{2 \tau}{d} \frac{6}{9 c_{0}-c_{1}} c_{6}^{2}\left(\tau^{2}+h^{4}\right)^{2}, \quad 0 \leq k \leq l .
\end{aligned}
$$

Summing up (45) for $k$, noticing (33), and exploiting (26), we have

$$
\begin{aligned}
\left|e^{k+1}\right|_{1}^{2} \leq & \left|e^{0}\right|_{1}^{2}+\frac{2 \tau}{d} \frac{30}{9 c_{0}-c_{1}} c_{3}^{2} \\
& \times \sum_{m=0}^{k}\left(\left\|e^{m}\right\|^{2}+\left\|e^{m-1}\right\|^{2}\right)+\frac{2 \tau}{d} \frac{6}{9 c_{0}-c_{1}} c_{4}^{2} \\
& \times \sum_{m=0}^{k}\left(\left\|e^{m+1-j}\right\|^{2}+\left\|e^{m-j}\right\|^{2}\right) \\
& +\frac{2 \tau}{d} \frac{6}{9 c_{0}-c_{1}} c_{6}^{2} \sum_{m=0}^{l}\left(\tau^{2}+h^{4}\right)^{2} \\
\leq & \frac{24\left(5 c_{3}^{2}+c_{4}^{2}\right)}{d\left(9 c_{0}-c_{1}\right)} \tau \sum_{m=1}^{k}\left\|e^{m}\right\|^{2}+\frac{12 c_{6}^{2}(l+1) \tau}{d\left(9 c_{0}-c_{1}\right)}\left(\tau^{2}+h^{4}\right)^{2} \\
\leq & \frac{4\left(5 c_{3}^{2}+c_{4}^{2}\right)}{d\left(9 c_{0}-c_{1}\right)} \tau \\
& \times \sum_{m=1}^{k}\left|e^{m}\right|^{2}+\frac{12 c_{6}^{2} T}{d\left(9 c_{0}-c_{1}\right)}\left(\tau^{2}+h^{4}\right)^{2}, \quad 0 \leq k \leq l .
\end{aligned}
$$

By Lemma 2, we have

$$
\left|e^{l+1}\right|_{1}^{2} \leq \frac{12 c_{6}^{2} T}{d\left(9 c_{0}-c_{1}\right)} \exp \left(\frac{4\left(5 c_{3}^{2}+c_{4}^{2}\right)}{d\left(9 c_{0}-c_{1}\right)} T\right)\left(\tau^{2}+h^{4}\right)^{2} .
$$

TABLE 1: Numerical results of $(54)$ when $h=1 / 10, \tau=1 / 100$.

\begin{tabular}{lccc}
\hline$(x, t)$ & $\begin{array}{c}\text { Numerical } \\
\text { solution }\end{array}$ & $\begin{array}{c}\text { Exact } \\
\text { solution }\end{array}$ & $\left|u\left(x_{i}, t_{k}\right)-u_{i}^{k}\right|$ \\
\hline$(0.5,0.1)$ & 0.667184 & 0.667184 & $8.652 e-009$ \\
$(0.5,0.2)$ & 0.727837 & 0.727837 & $1.623 e-008$ \\
$(0.5,0.3)$ & 0.788490 & 0.788490 & $2.258 e-008$ \\
$(0.5,0.4)$ & 0.849143 & 0.849143 & $2.805 e-008$ \\
$(0.5,0.5)$ & 0.909796 & 0.909796 & $3.288 e-008$ \\
$(0.5,0.6)$ & 0.970449 & 0.970449 & $3.725 e-008$ \\
$(0.5,0.7)$ & 1.031102 & 1.031102 & $4.129 e-008$ \\
$(0.5,0.8)$ & 1.091755 & 1.091755 & $4.509 e-008$ \\
$(0.5,0.9)$ & 1.152408 & 1.152408 & $4.870 e-008$ \\
$(0.5,1.0)$ & 1.213061 & 1.213061 & $5.218 e-008$ \\
\hline
\end{tabular}

From (25), we obtain

$$
\left\|e^{l+1}\right\|_{\infty} \leq c_{6} \sqrt{\frac{3 T}{d\left(9 c_{0}-c_{1}\right)}} \exp \left(\frac{2\left(5 c_{3}^{2}+c_{4}^{2}\right)}{d\left(9 c_{0}-c_{1}\right)} T\right)\left(\tau^{2}+h^{4}\right) .
$$

By the inductive principle, this completes the proof.

To discuss the stability of the difference scheme (20)-(22), we consider the following problem:

$$
\begin{gathered}
r(x, t) v_{t}-d v_{x x}=f(v(x, t), v(x, t-s), x, t), \\
(x, t) \in(0,1) \times(0, T], \\
v(x, t)=\phi(x, t)+\psi(x, t), \quad x \in[0,1], t \in[-s, 0], \\
v(0, t)=\alpha(t), \quad v(1, t)=\beta(t), \quad t \in(0, T],
\end{gathered}
$$

where $\psi(x, t)$ is the perturbation caused by $\phi(x, t)$. The following difference scheme solving for (49) can be obtained:

$$
\begin{gathered}
\mathscr{A} r_{i}^{k+(1 / 2)} \delta_{t} v_{i}^{k+(1 / 2)}-d \delta_{x}^{2} v_{i}^{k+1 / 2} \\
=\mathscr{A} f\left(\frac{3}{2} v_{i}^{k}-\frac{1}{2} v_{i}^{k-1}, \frac{1}{2} v_{i}^{k+1-j}+\frac{1}{2} v_{i}^{k-j}, x_{i}, t_{k+(1 / 2)}\right), \\
\quad 1 \leq i \leq M-1,0 \leq k \leq N-1, \\
v_{i}^{k}=\phi\left(x_{i}, t_{k}\right)+\psi_{i}^{k}, \quad 0 \leq i \leq M,-j \leq k \leq 0, \\
v_{0}^{k}=\alpha\left(t_{k}\right), \quad v_{M}^{k}=\beta\left(t_{k}\right), \quad 1 \leq k \leq N .
\end{gathered}
$$

Similar to the proof of Theorem 4 , the following stability result can be obtained

\section{Theorem 5. Denote}

$$
\eta_{i}^{k}=v_{i}^{k}-u_{i}^{k}, \quad 0 \leq i \leq M,-j \leq k \leq N .
$$

Then, there exist constants $\mathcal{c}_{7}$ and $c_{8}$ such that

$$
\left\|\eta^{k}\right\|_{\infty} \leq c_{7} \sqrt{\tau h \sum_{m=-j}^{0} \sum_{i=l}^{M-1}\left(\psi_{i}^{k}\right)^{2}}
$$

under the condition that $h$ and $\tau$ are small enough and $\max _{-j \leq k \leq 0,0 \leq i \leq M}\left|\psi_{i}^{k}\right| \leq c_{8}$. 
TABLE 2: Maximum norm errors of (54) with different step-sizes.

\begin{tabular}{lccc}
\hline$h$ & $\tau$ & $E_{\infty}(h, \tau)$ & $E_{\infty}(h, \tau) / E_{\infty}(h / 2, \tau / 2)$ \\
\hline $1 / 10$ & $1 / 100$ & $5.220 e-008$ & $*$ \\
$1 / 20$ & $1 / 400$ & $3.295 e-009$ & 15.842 \\
$1 / 40$ & $1 / 1600$ & $2.059 e-010$ & 15.999 \\
$1 / 80$ & $1 / 6400$ & $1.283 e-011$ & 16.046 \\
$1 / 160$ & $1 / 25600$ & $6.566 e-013$ & 19.546 \\
\hline
\end{tabular}

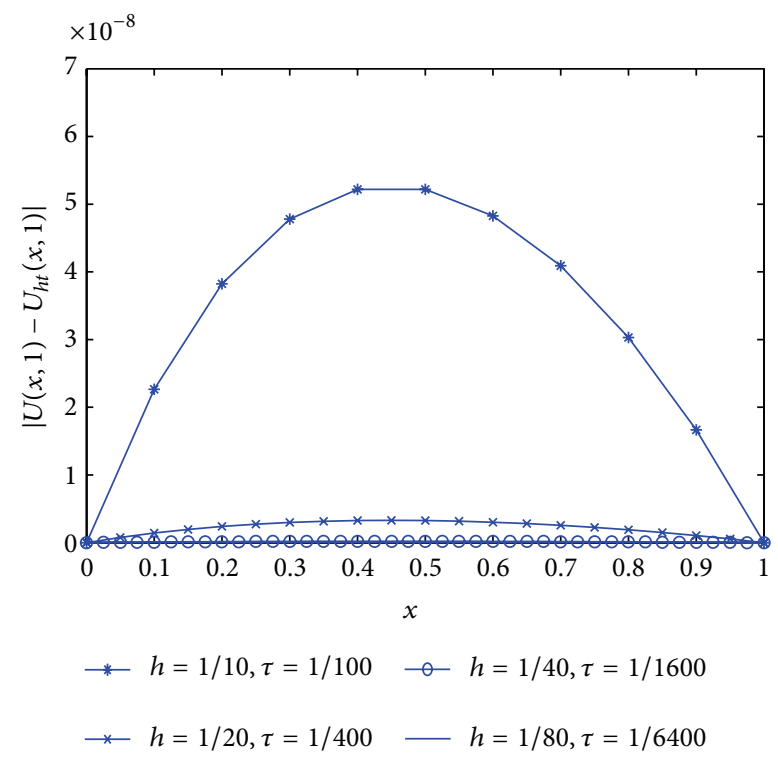

FIGURE 1: Error curves of difference scheme (20)-(22) solving for problem (54) with different step-sizes when $t=1$.

\section{Numerical Test}

In this section, a numerical test is considered to validate the algorithms provided in this paper, and the numerical solutions $u_{i}^{k}$ of the example are obtained by exploiting scheme (20)-(22).

Define

$$
E_{\infty}(h, \tau)=\max _{0 \leq i \leq M, 0 \leq k \leq N}\left|u\left(x_{i}, t_{k}\right)-u_{i}^{k}\right| .
$$

Example 1. Consider the following problem:

$$
\begin{gathered}
r(x, t) u_{t}-2 u_{x x}=u(x, t)+u(x, t-0.1)+2 e^{-x}, \\
x \in(0,1), t \in(0,1], \\
u(x, t)=e^{-x}(1+t), \quad x \in(0,1), t \in[-0.1,0], \\
u(0, t)=1+t, \quad u(1, t)=e^{-1}(1+t), \quad t \in(0,1],
\end{gathered}
$$

where $r(x, t)=4 t+5.9$. The exact solution of $(54)$ is $u(x, t)=$ $e^{-x}(1+t)$.

Table 1 provides some numerical results of difference scheme (20)-(22) solving for (54) with step-sizes $h=1 / 10$ and $\tau=1 / 100$. Table 2 gives the maximum absolute errors between numerical solutions and exact solutions with

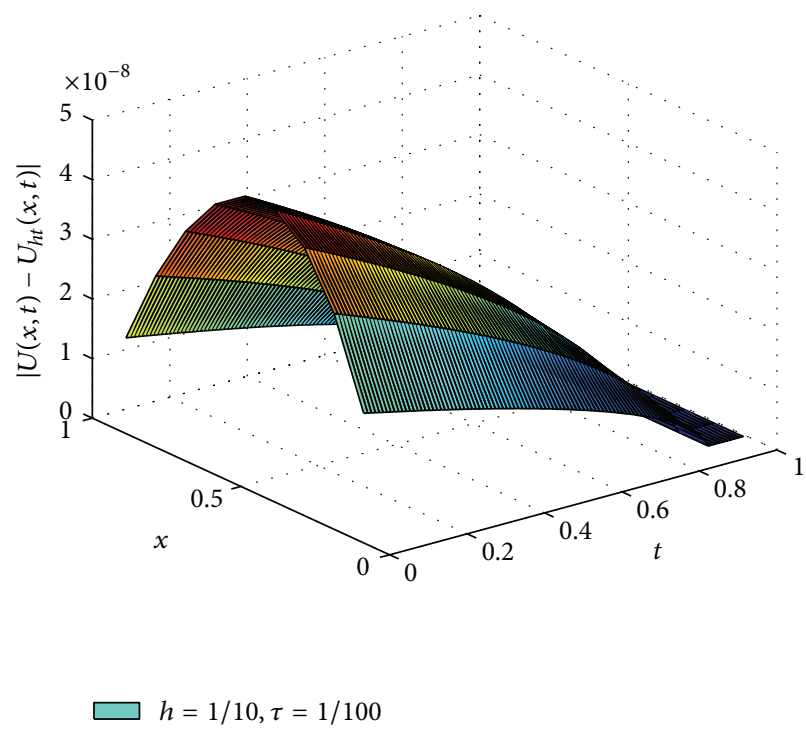

FIGURE 2: Error surface maps of difference scheme (20)-(22) solving for problem (54) with step-size $h=1 / 10 ; \tau=1 / 100$.

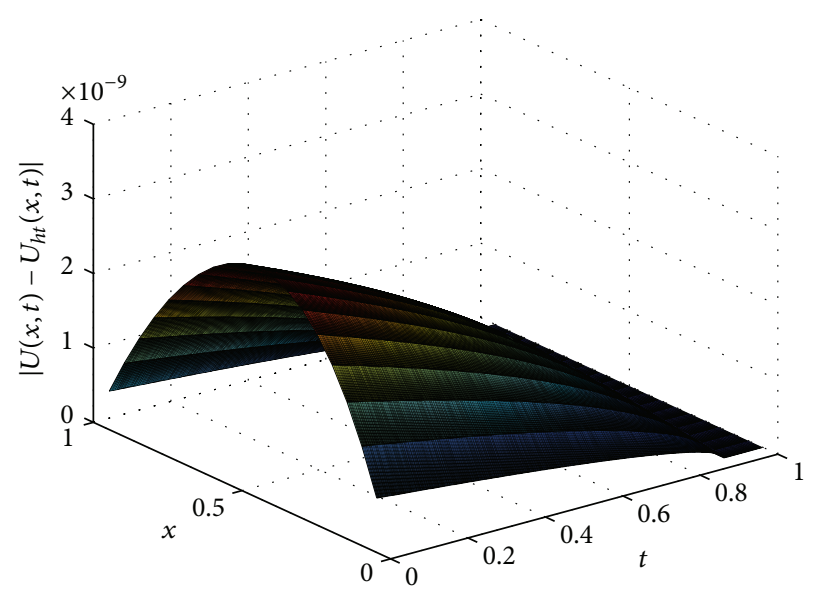

$\square h=1 / 20, \tau=1 / 400$

FIGURE 3: Error surface maps of difference scheme (20)-(22) solving for problem (54) with step-size $h=1 / 20 ; \tau=1 / 400$.

different step-sizes. From Table 2, we can see that when the space step-size and the time step-size are reduced by a factor of $1 / 2$ and $1 / 4$, respectively, then the maximum absolute errors are reduced by a factor of approximately $1 / 16$.

Figure 1 provides us the error curves of numerical solutions for (54) at $t=1$ by using scheme (20)-(22). Figures 2 and 3 give the error surface of the numerical solutions with step-sizes $h=1 / 10, \tau=1 / 100$, and $h=1 / 20, \tau=1 / 400$, respectively.

Generally speaking, from the results of the tables and the figures provided, we can see that the numerical results are coincident with the theoretical results. 


\section{Conclusion}

In this paper, a compact difference scheme is constructed to solve a type of variable coefficient delay partial differential equations, and the difference scheme is proved to be unconditionally stable and convergent. Finally, a numerical test is presented to illustrate the theoretical results.

\section{Conflict of Interests}

The author declares that there is no conflict of interests regarding the publication of this paper.

\section{Acknowledgments}

This work is supported by the Project Sponsored by the Scientific Research Foundation for the Returned Overseas Chinese Scholars, State Education Ministry (no. 2013693) and the National Natural Science Foundation of China (nos. 71301166, 11301544, and 11201487).

\section{References}

[1] J. H. Wu, Theory and Application of Partial Functional Differential Equation, Springer, New York, NY, USA, 1996.

[2] A. Bellen and M. Zennaro, Numerical Methods for Delay Differential Equations, Oxford University Press, Oxford, UK, 2003.

[3] H. Brunner, Collocation Methods for Volterra Integral and Related Functional Differential Equations, Cambridge University Press, Cambridge, UK, 2004.

[4] D. Li and C. Zhang, " $\mathrm{L}^{\infty}$ error estimates of discontinuous Galerkin methods for delay differential equations," Applied Numerical Mathematics, vol. 82, pp. 1-10, 2014.

[5] H. R. Marzban and H. R. Tabrizidooz, "A hybrid approximation method for solving Hutchinson's equation," Communications in Nonlinear Science and Numerical Simulation, vol. 17, no. 1, pp. 100-109, 2012.

[6] Z. Jackiewicz and B. Zubik-Kowal, "Spectral collocation and waveform relaxation methods for nonlinear delay partial differential equations," Applied Numerical Mathematics, vol. 56, no. 3-4, pp. 433-443, 2006.

[7] A. R. Ansari, S. A. Bakr, and G. I. Shishkin, "A parameterrobust finite difference method for singularly perturbed delay parabolic partial differential equations," Journal of Computational and Applied Mathematics, vol. 205, no. 1, pp. 552-566, 2007.

[8] D. Li, C. Zhang, and W. Wang, "Long time behavior of nonFickian delay reaction-diffusion equations," Nonlinear Analysis: Real World Applications, vol. 13, no. 3, pp. 1401-1415, 2012.

[9] F. A. Rihan, "Computational methods for delay parabolic and time-fractional partial differential equations," Numerical Methods for Partial Differential Equations, vol. 26, no. 6, pp. 1556-1571, 2010.

[10] D. Li, C. Zhang, and H. Qin, "LDG method for reactiondiffusion dynamical systems with time delay," Applied Mathematics and Computation, vol. 217, no. 22, pp. 9173-9181, 2011.

[11] D. Li and C. Zhang, "On the long time simulation of reactiondiffusion equations with delay," The Scientific World Journal, vol. 2014, Article ID 186802, 5 pages, 2014.
[12] D. Li, C. Tong, and J. Wen, "Stability of exact and discrete energy for non-fickian reaction-diffusion equations with a variable delay," Abstract and Applied Analysis, vol. 2014, Article ID 840573, 9 pages, 2014.

[13] H. Tian, "Asymptotic stability of numerical methods for linear delay parabolic differential equations," Computers \& Mathematics with Applications, vol. 56, no. 7, pp. 1758-1765, 2008.

[14] J. A. Ferreira and P. M. da Silva, "Energy estimates for delay diffusion-reaction equations," Journal of Computational Mathematics, vol. 26, no. 4, pp. 536-553, 2008.

[15] Z. B. Zhang and Z. Z. Sun, "A Crank-Nicolson scheme for a class of delay nonlinear parabolic differential equations," Journal on Numerical Methods and Computer Applications, vol. 31, no. 2, pp. 131-140, 2010.

[16] Z.-Z. Sun and Z.-B. Zhang, "A linearized compact difference scheme for a class of nonlinear delay partial differential equations," Applied Mathematical Modelling, vol. 37, no. 3, pp. 742752, 2013.

[17] Q. Zhang and C. Zhang, "A new linearized compact multisplitting scheme for the nonlinear convection-reaction-diffusion equations with delay," Communications in Nonlinear Science and Numerical Simulation, vol. 18, no. 12, pp. 3278-3288, 2013.

[18] W. Gu and P. Wang, "A Crank-Nicolson difference scheme for solving a type of variable coefficient delay partial differential equations," Journal of Applied Mathematics, vol. 2014, Article ID 560567, 6 pages, 2014.

[19] Z.-Z.Sun, The Numerical Methods for Partial Equations, Science Press, Beijing, China, 2005, Chinese.

[20] Z.-Z. Sun, "Compact difference schemes for heat equation with Neumann boundary conditions," Numerical Methods for Partial Differential Equations, vol. 25, no. 6, pp. 1320-1341, 2009. 


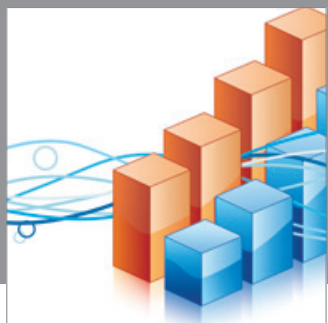

Advances in

Operations Research

mansans

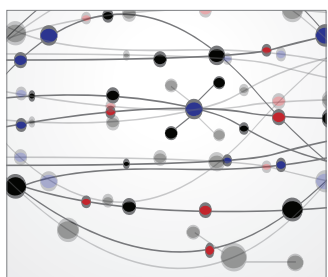

The Scientific World Journal
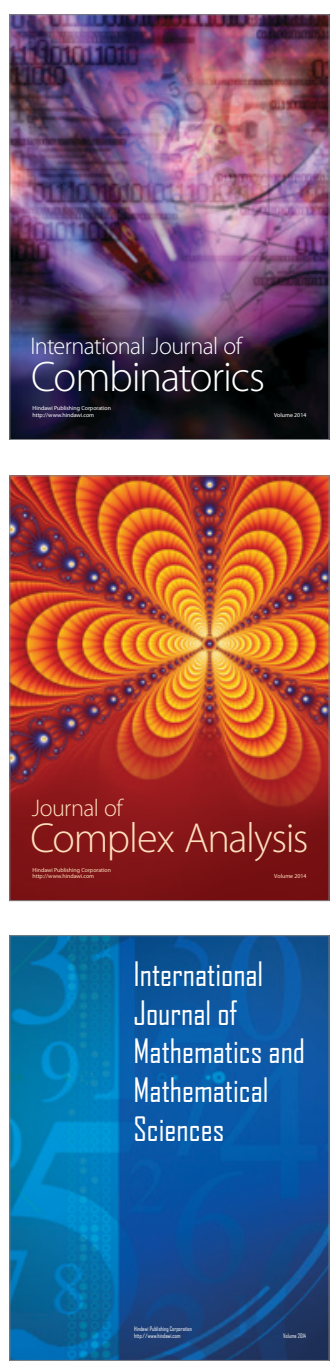
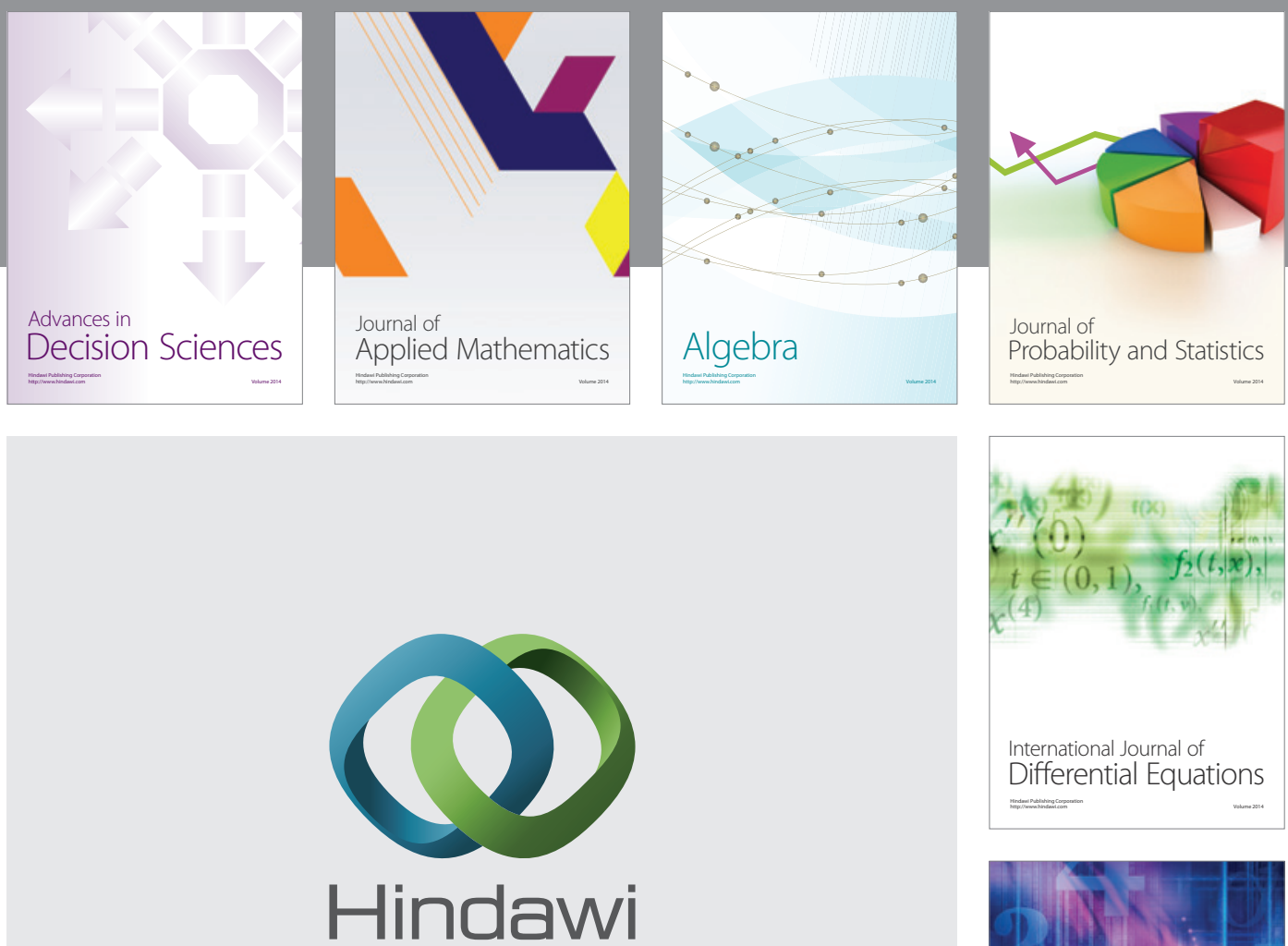

Submit your manuscripts at http://www.hindawi.com
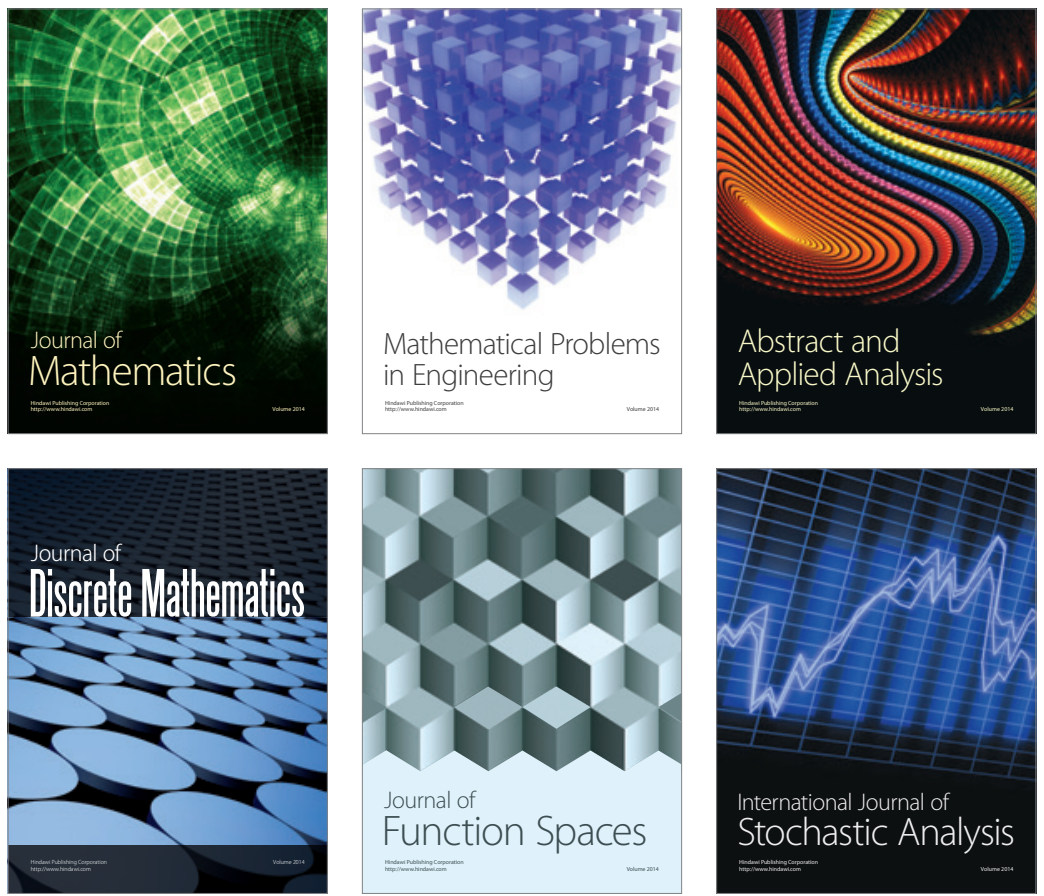

Journal of

Function Spaces

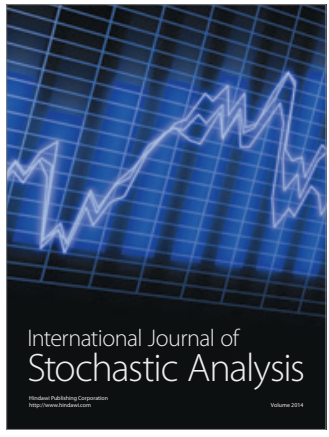

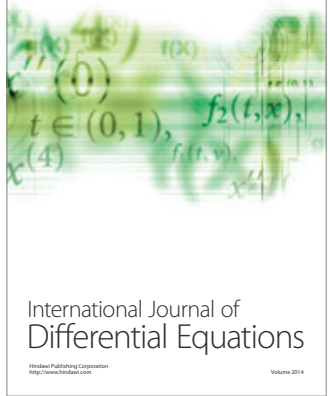
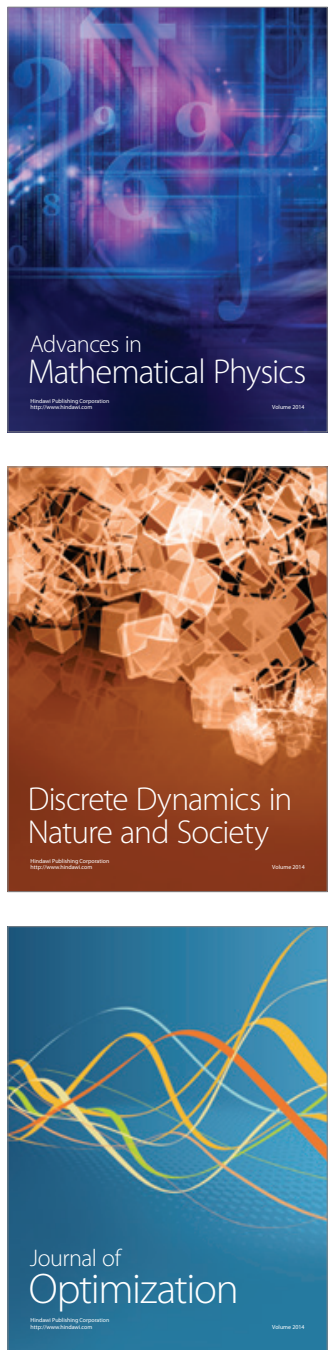